\title{
Research on Questioning Strategies of Senior Class Teachers in Kindergartens
}

\author{
Lina Zhang Rui Zhao \\ Teachers' College Shenyang University \\ Shenyang, China \\ Teachers' College Shenyang University \\ Shenyang, China
}

\section{Keywords: Senior class teachers; Questioning strategies; Teacher-child interaction}

\begin{abstract}
Questioning as a bridge between individuals in daily life, refers to individuals integrating into groups, not only a simple traditional concept and framework that you ask me to answer, such as "teacher-student" and "leadership-execution". The article put forward questions first and analyzes the reasons for the existence of the problem from subjective factors and objective factors. Finally, five aspects are proposed to solve the problem that are to respect the physical and mental development characteristics of the children in senior classes, and to mobilize the initiative of young children to discover and explore; to give play to the educational wisdom of kindergarten teachers and pay attention to the timing of questioning and the choice of objects; to eliminate invalid questions and prepare questions adequately; in the process of asking questions, trying to reflect fairness and focus on each child's performance, and improve the effectiveness of the question feedback.
\end{abstract}

\section{Presentation of questions}

"Guidance outline for kindergarten education (trial)", "Guidelines for 3-6 years old children's learning and development", "National medium- and long-term reform and development outline" in 2010 and "Starting the legislation of preschool education law" in 2017 require teachers to actively promote the physical and mental development of young children and implement their aim in the design of each activity. Questioning as an important means for teachers to pass on values, knowledge, experience, is a manifestation of the teaching process, which plays an important role. American psychologist Bruner once pointed out, "the teaching process is a continuous activity to put forward questions and solve problems." ${ }^{11]}$ Among them, effective questioning is an indirect expression of the education concept for kindergarten senior classes. It is an important link in the teaching process for kindergarten senior classes, and inserted into the design target, content, organization, implementation, evaluation of this organic system in each activity. According to the characteristics of each child's physical and mental development, the initiative of young children is mobilized to discover and explore, enlightening their thinking.

At present, the teachers have different levels of teacher specialization in each kindergarten senior classes. In the kindergarten activities based on the interaction of collective activities, the current status that the teachers put forward questions using the closed mode to question and answer with habitual pet phrases, makes the children really participating in the activities very inefficiently. Many scholars have pointed out that teachers should accelerate to promote self-professional development, including their own knowledge reserves, the renewal of educational concepts, and equal treatment to each child with an equal respect attitude. This requires us as teachers to think about how to ask questions in line with the current needs of the young children in senior classes, their physical and mental development, how to ask questions to be innovative, novel, attract their attention and can be maintained for a long time, which are the current concerns of scholars at home and abroad. 


\section{Factors affecting the questioning behaviors of kindergarten teachers in senior classes}

Effective questioning is an indirect expression of the education concept for kindergarten senior classes. The questioning behaviors of kindergarten teachers in senior classes is influenced by both subjective and objective factors.

(1) Subjective factors

The educational concept of kindergarten teachers in senior classes largely restricts the questioning behavior and teaching effect, directly influences the way and strategy of questioning, and determines the direction of education and teaching activities. All of these require teachers to establish correct concepts of education and questioning.

The attention degree of the kindergarten teachers in senior classes to the children affects the teachers in questioning behaviors. Joseph Chiari said, "The development of a person depends on the development of all people who interact directly and indirectly with him." In kindergartens, children in all activities are contacted by teachers. It is necessary for teachers to be able to understand the basic situation of different children, including the degree of communication with parents, the changes of children, and these entry points to questions will make the children feel that the teacher knows "I", "I" am here, and "I" am also an important role. In the research by Brophy Good pointed out that the attitudes of teachers also directly affect the teacher-child interaction. In the teacher-child interaction, the teachers do not like the children who are overactive and often have disciplinary problems. This part of the children is less concerned by the teachers, while those who are close to the teacher and disciplined are often concerned by the teachers. To achieve a fair starting point in education and promote the development of each child, teachers need to have a basic rational judgment on the basic development of young children in their classes.

(2) Objective factors

(i) The current development level of children affects the questioning behaviors of kindergarten teachers in senior classes

From the perspective of constructivist learning, Piaget put forward the theory of "zone of proximal development". Senior-class children are in the pre-operational stage, whose thinking logic of development is mainly based on the image in consciousness and self-feeling, with the external performance characteristics to treat the reasoning of external things. Thus the "daily concept" and the "scientific concept" in learning are continuously "shuffled" to build their own knowledge system. In the process of integrating new and old knowledge, the different development levels of each child will affect the questioning behaviors that are preset and created by the teachers in educational and teaching activities for children development. When the questioning behaviors of the kindergarten teachers acquire the children's thinking, they will experience the learning pleasure brought by the questions, which will promote the effective transformation inside the children and improve the quality of teaching.

(ii) Teacher-child interaction is the bond of effective questioning behaviors

The theory of symbolic interaction holds that symbols as a medium of interpersonal interaction are constructed for the individual; ${ }^{2]}$ different interactions produce different individual minds and also form different social outcomes and changes. This theory is of great significance to the study of teacher-child relationship in classroom questioning. The intimate degree of the relationship between teachers and children will indirectly affect the teachers' attention to different children, reflecting in whether there is physical contact, such as holding hands, hugging, touching, teachers' daily care for young children.

The teachers need to deliberately create a good atmosphere, cultivate a tacit understanding with young children, and ensure discipline restraint and positive responses form young children when performing their questioning behaviors.

(iii) Creating environmental quality is an important factor in teachers questioning behaviors

Class environment creation as an important component factor to evaluate the quality of kindergarten education, edifies the aesthetics and values of children. An environment with the characteristics of young children development can make children feel comfortable at the time and concentrate the children's attention. Activities implementation based on such a harmonious 
atmosphere requires teachers to think hard to better fulfill the teaching objectives, blending in the questioning behavior better with the environment to attract the attention of the children.

(3) Strategies for effective questioning by kindergarten teachers in senior classes

As a resource in the kindergarten senior classes, questioning is ubiquitous. Only by exploring the real needs of young children and carrying out targeted treatment can be improved the quality of children reactions, to fully realize the rational allocation of resources and achieve educational equity [3].

(i) Respect senior-class children in their physical and mental development characteristics, and mobilize the initiative of young children to discover and explore

The "Outline" points out that questioning as an important means to complete the teaching task, can reasonably, effectively, and scientifically stimulate the interest of young children, promote the development of children's thinking and creativity, receiving the expected teaching effect. An effective question can connect icons in different coordinates to each other, constantly dig into the entry point of the children psychological world to their daily life, so that the child has the capacity to think about the problem, actively exploring the question with the teacher and the child. These are the manifestations of subjectivity, that is, both are the main body of education and teaching activities. Teachers create a free, relaxing and sustained dialogue on the premise of respecting the physical and mental development of young children, meanwhile actively encourage children to express their emotions, and continue to help young children initiatively construct the zone of proximal development in education and teaching activities.

(ii) Give play to the educational wisdom of kindergarten teachers, and pay attention to the timing of questioning and the selection of objects

With the continuous improvement of teachers' professional level, professional self-identity of the teachers has been continuously improved, and their teaching confidence has been continuously enhanced. As the most direct kindergarten teachers who communicate with children in the first line, they have a wealth of practical experience. The passing way in which teachers complete the goal of teaching activities is mainly driven by questioning., but the continuity of questioning is very poor. Often the feedbacks from teachers is affirmative or directly negating the children's answers with two or three sentences, and the teachers will instinctively ask those children who are actively performing, reflecting a phenomenon that the current kindergarten teachers in senior classes have low overall questioning levels. The expansion space of the questioning ability is still not fully exerted.

(iii) Eliminate invalid questioning, and prepare questions adequately

For the kindergarten teachers in senior classes, a delicate question is profited from the teacher's full understanding of the "guidelines", the internal relationship between the children's existing knowledge and experience and the content of the courses, and preparedly thinking about how to make young children think, imagine and express their emotions in a limited time and space, before the theme activities in each field. Teachers need to repeatedly return to the theme in promoting the children's divergent thinking and answering during the activity, avoid deviation from the theme, rationally arrange the difficulty of the problem when designing the questions, and make"differential treatment" for the children at different levels, prompting the children at different levels to achieve the optimum at their own level through asking questions.

(iv) In the process of questioning, try to embody fairness and pay attention to the performance of each child.

The teaching method of kindergartens in China is mainly collective teaching. The questioning target can be all students or a certain student. Liu Chengyu and Guo Boxiang's statistics data show that in addition to the collective questioning in the teaching process, $84 \%$ of the questions are directed at students with strong abilities, and many students are only in the state of "accompanied to sit". ${ }^{[4]}$ A question that has a positive developmental effect on young children should be used reasonably during the questioning process, focusing on the performance of the children questioning to the teachers, which is embodied in the expression, posture, attention, etc. It is necessary to achieve special treatment and differential treatment, ${ }^{[5]}$ observe those children who are 
"accompanied to sit" in the questioning, utilize body language, hugs, handshakes, etc. to communicate with them more after classes, understand their psychological needs to find out the reason, and encourage them to express themselves, to achieve the development of fairness.

(v) Improve the effectiveness of question feedback

An excellent theme activity consists of a teacher who emits signals, the children who are receivers, and a teacher who reprocesses the signals. The teacher who reprocesses the signals as a link of questioning feedbacks serves as an important indicator to evaluate the quality of the theme activity. It is found through observing the activities that the feedback on the performance of young children is still in a state of exhaustion for the kindergarten teachers in senior classes, that is the praise and criticism of the so-called given pattern. When teachers making use of it, they usually combine the tone and facial expression to show their affirmation or negation to the children's practice, lack of pertinence and the reflection of teachers' emotion to the children, which cannot make young children feel that my performance today is better than before and no room for the children to think about their progressive space. The teacher's feedback to the children should not only purpose to implement the teaching task with simple feedbacks, and such teaching is also worthless. Whereas, effective guidance should be provided for young children to help them grow.

\section{Conclusions}

Questioning by the kindergarten teachers in senior classes serves as an important method and mean in collective teaching, connecting the development quality of teacher-child interaction. On the premise of respecting the development of young children, kindergarten teachers should pay attention to the development needs of each child and meet their own zone of proximal development, so that children can reach their best level. Effective questioning can actively develop the children in cognition, emotion and will, and make the children learn to express emotions and release emotions; for teachers, it is necessary to make reflective practice as the purpose of their own educational and teaching activities, constantly improve their own quality and professional development level, and reasonably allocate the questioning resources to achieve a fair starting point and ensure the overall quality of teaching. Meanwhile, invalid questions should be abandoned, and more open questions should be put forward to stimulate children's interest and inspire their thinking. "Teaching from doing, learning from doing" is regarded as the basic concept of education, to give full play to the collective wisdom of teachers, constantly optimize questioning and achieve high quality feedback.

\section{References}

[1] Wu Tingting. Effective Questioning Strategies of Picture Talk Activities in Kindergartens . [J]. Education and Teaching Research, 2013.

[2] Kang Taoxia. Research on Optimization of Teachers' Questioning Strategies in Group Teaching of Kindergartens [J]. South-west University, 2011.

[3] Liu Shuhui. The Creation of the Secure Environment for Infants through Family-kindergarten cooperation [J]. Guide to Science and Education, 2015(03):142-143.

[4] Yang Xiaoping, Li Min. The development of Preschool Education in the view of Justice and Quality[J]. Preschool Education Research, 2015, (06): 3-8.

[5] Liu Yanjin. Reconsideration of Teachers' Questioning Strategies in Kindergarten Teaching [J]. Curriculum Research, 2016, (03): 96-97. 